\title{
Contribution of QCD Condensates to the OPE of Green Functions of Chiral Currents
}

\section{Tomáš Kadavý*}

Institute of Particle and Nuclear Physics, Charles University, Prague, Czech Republic

E-mail: kadavy@ipnp.troja.mff.cuni.cz

\section{Karol Kampf}

Institute of Particle and Nuclear Physics, Charles University, Prague, Czech Republic Department of Astronomy and Theoretical Physics, Lund University, Lund, Sweden

E-mail: kampf@ipnp.troja.mff.cuni.cz

\section{Jiří Novotný}

Institute of Particle and Nuclear Physics, Charles University, Prague, Czech Republic

E-mail: novotny@ipnp.troja.mff.cuni.cz

\begin{abstract}
Correlators of the odd-intrinsic parity sector of QCD form a significant part of all Green functions of chiral currents. To calculate contribution of resonances to the correlators of the odd sector at NLO, one can use the resonance Lagrangian [3], which, however, still contains many unknown coupling constants. One of the ways of obtaining them is to compare the resonance contribution with the behaviour of such correlator at high energies. The operator product expansion (OPE) is a useful framework for such an approach. The matching leads to important coupling constants constraints that are expressed in terms of QCD condensates.
\end{abstract}

The 39th International Conference on High Energy Physics (ICHEP2018)

4-11 July, 2018

Seoul, Korea

${ }^{*}$ Speaker. 


\section{Introduction}

Green functions of chiral currents represent a tool of paramount importance since they can be used to calculate amplitudes of physical processes, with the help of the LSZ reduction formula. In our work, we are interested in the three-point Green functions constructed of the octet vector $V_{\mu}^{a}(x)=\bar{q}(x) \gamma_{\mu} T^{a} q(x)$ and axial-vector $A_{\mu}^{a}(x)=\bar{q}(x) \gamma_{\mu} \gamma_{5} T^{a} q(x)$ chiral currents or scalar $S^{a}(x)=$ $\bar{q}(x) T^{a} q(x)$ and pseudoscalar $P^{a}(x)=i \bar{q}(x) \gamma_{5} T^{a} q(x)$ densities, where the flavor matrices $T^{a}$ are defined as $T^{a}=\lambda^{a} / 2$ with $\lambda^{a}$ being the Gell-Mann matrices.

Generally, 15 non-trivial three-point Green functions (correlators) made of chiral currents and densities exist in QCD. As it turns out later, it is useful to separate them into two groups (we intentionally omit the Lorentz and flavor indices):

- Set I: $\langle A S P\rangle,\langle V S S\rangle,\langle V P P\rangle,\langle V V A\rangle,\langle A A A\rangle,\langle A A V\rangle,\langle V V V\rangle$.

- Set II: $\langle S S S\rangle,\langle S P P\rangle,\langle V V P\rangle,\langle A A P\rangle,\langle V A S\rangle,\langle V V S\rangle,\langle A A S\rangle,\langle V A P\rangle$.

A part of the Green functions above belong to the odd-intrinsic parity sector of QCD, namely $\langle V V A\rangle,\langle A A A\rangle,\langle V V P\rangle,\langle A A P\rangle$ and $\langle V A S\rangle$. Then, a contribution of resonances to these correlators can be studied within the Resonance chiral theory $(\mathrm{R} \chi \mathrm{T})[1,2]$, using the NLO resonance Lagrangian [3]

$$
\mathscr{L}_{\mathrm{R} \chi \mathrm{T}}^{(6)}=\sum_{X} \sum_{i} \kappa_{i}^{X}\left(\widehat{\mathscr{O}}_{i}^{X}\right)_{\mu v \alpha \beta} \varepsilon^{\mu v \alpha \beta},
$$

where $X$ stands for the resonance fields $V, A, S, P$, double-resonance fields $V V, A A, S A, S V, V A$, $P A, P V$ and triple-resonance fields $V V P, V A S, A A P$. This Lagrangian contains 67 operators and corresponding 67 unknown couplings $\kappa_{i}^{X}$. The specific form of the operator monomials $\left(\widehat{\mathscr{O}}_{i}^{X}\right)_{\mu v \alpha \beta}$ can be found in Ref. [3].

Many of the couplings $\kappa_{i}^{X}$ have not been determined yet. A one way to accomplish this is to study behaviour of Green functions at high energies. The operator product expansion (OPE) [4] is a suitable framework for such a task. It says that at large external momenta (or, in QCD, at short distances), a Green function can be written down as a sum of Wilson coefficients proportional to vacuum averages of composite gauge-invariant local operators made of quark and gluon fields, i.e. the QCD condensates. In other words, for all momenta large, or equivalently for $(x, y) \rightarrow(0,0)$, we are allowed to write

$$
\begin{aligned}
\left\langle\mathscr{O}_{1}(x) \mathscr{O}_{2}(y) \mathscr{O}_{3}(0)\right\rangle & =C_{\text {pert. }}+C_{\langle\bar{q} q\rangle}\langle\bar{q} q\rangle+C_{\left\langle G^{2}\right\rangle}\left\langle G_{\mu \nu} G^{\mu v}\right\rangle \\
& +C_{\langle\bar{q} G q\rangle}\left\langle\bar{q} \sigma_{\mu \nu} G^{\mu v} q\right\rangle+C_{\langle 4 q\rangle}\left\langle\bar{q} X_{1} q \bar{q} X_{2} q\right\rangle+\ldots
\end{aligned}
$$

The first term in (1.2) stands for the perturbative contribution and the subsequent ones represent the quark, gluon, quark-gluon and four-quark condensates. The Wilson coefficients $C_{i}$ contain information about short-distance physics, i.e. the dynamics above the scale $\mu$, and are calculable in perturbative QCD by the means of Feynman diagrams.

As it turns out, the correlators of the Set I have non-trivial perturbative contribution, followed by the contributions of the gluon and four-quark condensates. On the other hand, the quark and quark-gluon condensates contribute to the Green functions of the Set II. 


\section{Matching $\mathbf{R} \chi \mathrm{T}$ and $\mathrm{OPE}$}

To illustrate the matching of the $\mathrm{R} \chi \mathrm{T}$ and OPE, we chose the $\langle V V A\rangle$ Green function, which has been an object with a significant theoretical and phenomenological importance for a long time.

First of all, we performed a calculation [5] of this correlator using the resonance Lagrangian (1.1). Then, we tried to take an advantage of the result for OPE of $\left\langle V^{\star} V A\right\rangle$, where one of the momenta is soft [6]. Among the results of this matching is a prediction for the value of the deviation parameter $\delta_{\mathrm{BL}}$, which describes by how much is the Brodsky-Lepage behaviour of the pion transition formfactor $\mathscr{F}_{\pi^{0} \gamma \gamma}^{\mathrm{R} \chi \mathrm{T}}$ violated. We found $\delta_{\mathrm{BL}}=-1.342$. This value, however, is not compatible with the data sets for the measured pion transition formfactor, obtained by the BABAR [7], BELLE [8] and CLEO [9] collaborations. Therefore, using the OPE with one soft momentum does not lead to an agreement with experiments. Nevertheless, there is no argument that this kind of OPE would be the best choice to use.

We tried to solve this by calculating the OPE with all three momenta large, and study not only the $\langle V V A\rangle$ Green function, but every non-trivial three-point correlator. After revisiting the results of the quark condensate both at tree [10] and one-loop level [11], we found respective contributions of the gluon, quark-gluon and four-quark condensates to the Green functions of the Set I and II, as mentioned above [12].

The contributions of the quark-gluon and four-quark condensates are worth discussing in more detail. In fact, they contribute effectively, through a propagation of the non-local quark and quarkgluon condensates. To see this, one may use the Fock-Schwinger gauge [13] and after expanding the quark and gluon fields, it is apparent that such propagation can be written down as follows [12]:

$$
\begin{aligned}
\left\langle\bar{q}_{i, \alpha}^{A}(x) q_{k, \beta}^{B}(y)\right\rangle & =\left(\frac{1}{36}\langle\bar{q} q\rangle \delta_{i k}+\langle\bar{q} G q\rangle\left[F^{\langle\bar{q} q\rangle}(x, y)\right]_{k i}+\langle\bar{q} q\rangle^{2}\left[G^{\langle\bar{q} q\rangle}(x, y)\right]_{k i}\right) \delta_{\alpha \beta} \delta^{A B}, \\
g_{s}\left\langle\bar{q}_{i, \alpha}^{A}(x) \mathscr{A}_{\mu}^{a}(y) q_{k, \beta}^{B}(z)\right\rangle & =\left(\langle\bar{q} G q\rangle\left[F_{\mu}^{\langle\bar{q} \mathscr{A} q\rangle}(x, y, z)\right]_{k i}+\langle\bar{q} q\rangle^{2}\left[G_{\mu}^{\langle\bar{q} \mathscr{A} q\rangle}(x, y, z)\right]_{k i}\right)\left(T^{a}\right)_{\beta \alpha} \delta^{A B},
\end{aligned}
$$

where we assumed a validity of the dominance of an intermediate vacuum states in the large $N_{c}$ limit for the four-quark condensate. The functions $F$ and $G$ in (2.1)-(2.2) contain the coordinate dependance and their form can be found in Ref. [12].

To provide an illustration, the contributing topologies to the four-quark condensate are depicted below with a detailed description.
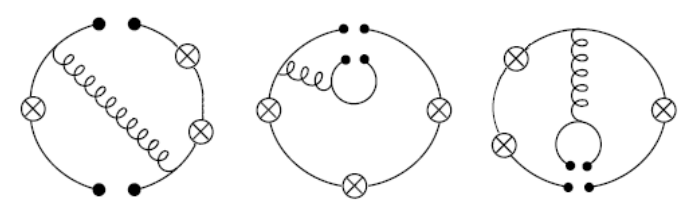

Figure 1: Feynman diagrams of contributions of the four-quark condensate to the three-point correlators. Left: this topology is given perturbatively since the exchanged gluon is hard. Middle: an effective propagation of the non-local quark condensate, given by (2.1) with the part proportional to $G^{\langle\bar{q} q\rangle}(x, y)$. Right: propagation of the non-local quark-gluon condensate, given by (2.2) with the part proportional to $G_{\mu}^{\langle\bar{q} \mathscr{A} q\rangle}(x, y, z)$. 


\section{Conclusion}

To conclude, we calculated OPE of all three-point Green functions of chiral currents for all momenta large, i.e. we expressed these correlators in terms of QCD condensates. Then we tried to match the OPE with $\mathrm{R} \chi \mathrm{T}$ again, however, it is still unclear how to deal with logarithmic terms for which one would need an infinite tower of resonances. The final results of our work will be available fairly soon in Ref. [5, 12].

\section{Acknowledgement}

TK wishes to thank the organizers for a very enjoyable conference. The work was supported by The Czech Science Foundation (project GACR no. 18-17224S) and The Ministry of Education, Youth and Sports of the Czech Republic (project MSMT no. LTT17018).

\section{References}

[1] G. Ecker, J. Gasser, A. Pich and E. de Rafael, The Role of Resonances in Chiral Perturbation Theory, Nucl. Phys. B 321 (1989) 311.

[2] V. Cirigliano, G. Ecker, M. Eidemuller, R. Kaiser, A. Pich and J. Portoles, Towards a consistent estimate of the chiral low-energy constants, Nucl. Phys. B 753 (2006) 139 [hep-ph/0603205].

[3] K. Kampf and J. Novotny, Resonance saturation in the odd-intrinsic parity sector of low-energy QCD, Phys. Rev. D 84 (2011) 014036 [hep-ph/1104.3137].

[4] K. G. Wilson, The Renormalization Group and Strong Interactions, Phys. Rev. D 3 (1971) 1818.

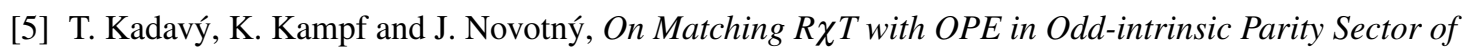
$Q C D$ (in preparation).

[6] P. Colangelo, F. De Fazio, J. J. Sanz-Cillero, F. Giannuzzi and S. Nicotri, Anomalous $A V^{*} V$ vertex function in the soft-wall holographic model of QCD, Phys. Rev. D 85 (2012) 035013 [hep-ph/1108.5945].

[7] B. Aubert et al. [BaBar Collaboration], Measurement of the gamma gamma* $\rightarrow$ pi0 transition form factor, Phys. Rev. D 80 (2009) 052002 [hep-ex/0905.4778].

[8] S. Uehara et al. [Belle Collaboration], Measurement of $\gamma \gamma^{*} \rightarrow \pi^{0}$ transition form factor at Belle, Phys. Rev. D 86 (2012) 092007 [hep-ex/1205.3249].

[9] J. Gronberg et al. [CLEO Collaboration], Measurements of the meson - photon transition form-factors of light pseudoscalar mesons at large momentum transfer, Phys. Rev. D 57 (1998) 33 [hep-ex/9707031].

[10] B. Moussallam, Chiral sum rules for parameters of the order six Lagrangian in the W-Z sector and application to piO, eta, eta-prime decays, Phys. Rev. D 51 (1995) 4939 [hep-ph/9407402].

[11] M. Jamin and V. Mateu, OPE-R(chi)T matching at order alpha(s): Hard gluonic corrections to three-point Green functions, JHEP 0804 (2008) 040 [hep-ph/0802.2669].

[12] T. Kadavý, K. Kampf and J. Novotný, On QCD Condensates and the Three-point Green Functions of Chiral Currents (in preparation).

[13] V. Fock, Proper time in classical and quantum mechanics, Phys. Z. Sowjetunion 12 (1937) 404. 